\title{
KONTEXTUELLE KONTINGEnZ: MUSIKCLIPS IM WISSENSCHAFTLICHEN UMGANG ${ }^{1}$
}

\author{
Christoph Jacke
}

\section{Einleitung}

In der letzten Zeit leuchtete das wissenschaftliche Scheinwerferlicht an den Musikclips $^{2}$ vorbei. Jüngst schrieb die Frankfurter Rundschau gar von der Bedrohung der Clips durch den "eigenen Akademismus« (Kothenschulte 2002: 23). Die Journalistin Fee Magdanz spricht von einem Verkommen der Popkultur zum Allgemeingut durch wissenschaftliche Substitutionsversuche ${ }^{3}$ :

"Es gibt Seminare über die Sprache des HipHop, über Techno-Moden, Musikvideokultur und natürlich Poptheorie, gepaart mit oder gar rudimentär resultierend aus der Fluktuation der Fragen der Gender und Cultural Studies. Nicht nur weil Pop aus sich selbst heraus die politischen Aspekte seiner Identität verworfen hat, sondern auch, weil er zu einer staubigen Theorie wurde, haben die gelebten Codes ihre Eindeutigkeit, den festen Standpunkt, von dem aus sie gesetzt wurden, und damit letztlich ihre Radikalität verloren « (Magdanz 2001: 133).

1 Ich danke den Studierenden, die an meiner Lehrveranstaltung »Exemplarische Kulturanalyse: Musikvideoclips - Kunst für die Massen?« an der Universität Münster im Sommersemester 2002 teilgenommen haben, für Anregungen und Diskussionsbereitschaft.

2 Im Weiteren verwende ich den Begriff Clip als Kürzel für medientechnologisch basierte und massenreproduzierte Musikkurzfilme. M.E. kann im Zeitalter der immer noch boomenden Internet-, CD ROM- und DVD-Rezeption nicht länger von Musikvideos geschrieben werden.

3 Magdanz ist insofern Recht zu geben, als dass das vor allem emphatische Erleben und Erfahren von Popkultur nicht zu verwissenschaftlichen ist; die Beobachtung dessen aber m.E. durchaus. Im übrigen: flüchten sich nicht einige der von Magdanz beschriebenen Popkulturzeitzeugen in Richtung Verwissenschaftlichung, um ihr genau dadurch (erneut) eine besondere, ggf. sogar zu musealisierende Bedeutung zuzuschreiben? 
Bei der Preisverleihung des MuVi-Preises der Oberhausener Kurzfilmtage 2002 schienen sich Juroren, Regisseure und Musiker seltsam einig, dass diese Auszeichnung keine besonderen Auswirkungen für die eigenen Laufbahnen hat und die Clips eher als Abfallprodukt anderer Tätigkeiten (z.B. Film- und Fernsehdokumentationen, Club-Visualisierungen) verstanden werden sollten. Zu diesem offensichtlichen Bedeutungsverlust scheint zu passen: Der Konsum von Musik, dementsprechend die Rezeption von Clips und Musikfernsehen im allgemeinen, wird im Datenreport 2002 des Statistischen Bundesamtes (2002) nicht erfasst.

Gleichzeitig lässt sich nicht übersehen, dass MTV und VIVA zu den bekanntesten Musiksender-Marken in Deutschland zählen und tägliche Publika erzeugen, von denen andere Formate nur träumen können (vgl. Kurp/Hauschild/Wiese 2002). Ferner liefen in den letzten Jahren mit Pop 2000 und den Fantastic Voyages zwei sehr beachtete und wiederholte Dokumentationen zu Popmusik und ihrer Visualisierung im deutschen Fernsehen. Als weiterer Indikator für die mediengesellschaftliche Aufmerksamkeit für Musikclips seien die durch die DVD- und Computertechnologien immer schneller und leichter zu produzierenden und rezipierenden Zusammenstellungen von Musikclips erwähnt. ${ }^{4}$ Lars Henrik Gass, Leiter der Internationalen Kurzfilmtage in Oberhausen, konstatiert sogar - ganz entgegen des Eindrucks von der MuVi-Awards-Preisverleihung - ein neu erwachtes Interesse an den Clips:

»Gerade die visuelle Seite der Musikvideos hat durch das Abtreten des Interpreten und seiner >Instrumente $<$ in der elektronischen Musik der letzten Jahre und durch die Wahrnehmung der enormen Imagepotenziale der Musikvideos einen ungeahnten Gestaltungsspielraum erhalten. Es gibt hier so etwas wie eine Anziehungskraft zwischen neuen Klangwelten und ihrem visuellen Widerpart« (Gass 2001: 88).

Es ist offensichtlich an der Zeit, sich erneut und um so intensiver mit Musikclips zu beschäftigen.

Ein kurzer Blick auf die Geschichte wissenschaftlicher Behandlungen von Clips soll zu der Frage führen, warum zunächst solcherlei Thematiken in den wissenschaftlichen Diskursen nur vereinzelt aufgegriffen wurden und eine fundierte Verbindung und Ergänzung verschiedener Standpunkte bzw. Disziplinen zu diesem Thema bis heute nur selten angewendet und umgesetzt wurde. Wie sollen etwa bei einer Produktanalyse Medienkulturwissenschaft-

4 Zwei aktuelle Beispiele für Kompilationen aus dem Bereich nicht-kommerzieller Clips und Tracks auf DVD sind Various Artists - Visual Niches - Extraordinary Music Videos (E:Motion / EFA) und For Promotional Use Only - Musivideos aus dem Off (Bold); beide im Jahr 2002 veröffentlicht. 
ler einen Clip fundiert erforschen, ohne den Sound analysieren zu können? Wie sollen Musikwissenschaftler einen Song mit Clip sezieren, ohne die Bewegungsbilder bspw. filmanalytisch untersuchen zu können? Wie sollen und können intra-, inter- und vor allem transdisziplinär die Beziehungen zwischen Bildern und Tönen betrachtet werden?

In meinem Beitrag werde ich nach dem historischen Abriss eine mögliche Differenzierung der Analyseebenen des massenkommunikativen Prozesses Musikclip und des Kompaktbegriffs Musikclip vorschlagen, anhand derer sich die vornehmlich betroffenen Disziplinen zuordnen, koorientieren und somit größere, transdisziplinäre Forschungsprojekte vorbereiten können. Beispielhafte Fragestellungen solcher Projekte sollen anschließend aufgelistet werden, um dann ein vorläufiges Fazit zu ziehen.

\section{Clip-Geschichten}

»Die triviale Bilderfülle von heute einfach nur festzustellen, wie es kulturkritische Mode ist, ist selbst trivial, hat einen phobischen oder manischen Zug.« (Neubaur 2002: 1079)

Es wurde bereits vieles über die Visualisierung und Medialisierung der popkulturellen Lebenswirklichkeiten gesagt und geschrieben. Die oben zitierte Religionswissenschaftlerin Caroline Neubaur weist zum einen zu Recht darauf hin, dass die Rolle des medialisierten Bildes meistens nur beklagt, nicht aber analytisch durchdrungen wird. ${ }^{5}$ Zum anderen beziehen sich laut Neubaur die feuilletonistischen und wissenschaftlichen Lamenti zu pauschal auf die so genannte Bilderflut, da es doch offensichtlich allgemeiner um einen Überfluss an Informationen geht (vgl. Neubaur 2002: 1081). Dies ist m.E. nicht ganz richtig: Es gibt keinen aufmerksamkeitsökonomischen kognitiven Overload, es kann immer nur kognitiv soviel aus der Umwelt verarbeitet werden, wie eben verarbeitet werden kann. Jedes kognitive System ist sein eigener Gatekeeper. Was die meisten Autoren in diesem Zusammenhang beschreiben, ist ein Überfluss an Informationsangeboten bzw. ein Überangebot an Möglichkeiten, ein Kontingenz-Overload. Und diese Möglichkeiten von Bilderangeboten haben sich im Zusammenhang mit der Ausdifferenzierung des deutschen Mediensystems in den letzten knapp zwanzig Jahren deutlich

5 Allerdings klingt Neubaurs Aburteilung von Medienwissenschaftlern und Semiotikern als ahnungslose >Gucker und die damit einhergehende Einforderung kunstwissenschaftlicher Qualifizierungen zu verallgemeinernd, haben sich doch längst in allen drei Feldern Spezialisten für etwa Theorien des Bilds herausdifferenziert. 
vervielfacht. Wenn sich einst jugendliche Rezipienten eine Woche lang auf die neue Folge von Formel Eins freuten und vorbereiteten, um sihre< Idole via Clip oder via Fernsehauftritt zumeist einmalig bewundern zu können, gibt es heute eine kaum noch zu überschauende Angebotsmöglichkeitenvielfalt. Dies heißt kultürlich (sensu P. Janich) nicht, dass die Angebote selbst mannigfaltig sind. Aber darum soll es hier nicht gehen.

Mit der erwähnten Ausdifferenzierung der musikalischen Angebote und ihrer immer weiter verbreiteten Vermarktung gingen in den 1980er und 1990er Jahren auch erste wissenschaftliche Beobachtungen einher. Wegweisend sind unter den frühen Veröffentlichungen sicherlich die Beobachtungen von Veruschka Bódy und Peter Weibel (1987), die den Musikclip aus seinen Vorläufern der Videokunst heraus beschreiben und mit zahlreichen Gastautoren ausführliche Überblicke über den seinerzeit synchronen und vor allem diachronen Zusammenhang von Kunst- und Musikkultur liefern. Bereits Ende der Achtziger erkannte Weibel zudem entdramatisiert die Werbefunktion von Musikclips, indem er sie als visuelle Logos für akustische Logos, die Popsongs eben, bezeichnet (vgl. Weibel 1987b: 274). Gegenüber eher historischen Abrissen ${ }^{6}$ und Beiträgen zur Einordnung von Clips und allgemeiner Popmusik zwischen Kunst und Nichtkunst ${ }^{7}$ gibt es konkrete Analysen zu einzelnen Clips oder Künstlern ${ }^{8}$ und Rezeptionsanalysen'. In all diesen Ausführungen wird klar, wie schwer eine auf einen Aspekt beschränkte Untersuchung ist und aus welch unterschiedlichen Perspektiven und Disziplinen (also Kontexten) die Clips analysiert werden.

In ihren hilfreichen Überblicken mit ausführlichen Bibliographien kristallisieren Neumann-Braun/Barth/Schmidt (1997) bzw. Neumann-Braun/ Schmidt (1999) im Wesentlichen vier Themen- und Problembereiche zu Musikclips heraus, auf denen sich die oft zwischen Wissenschaft und Journalismus mäandernden Auseinandersetzungen bewegen:

- Videoclips zwischen Avantgarde und Populärkultur,

- Geschichte, Ökonomie und Produktion von visueller Musik und Musikvideo,

- Produktanalysen von Videoclips,

- Nutzung und Rezeption von Videoclips und Musikfernsehen.

6 Vgl. statt anderer Langhoff 1999, Morgenroth 1994, Weibel 1987a, Winter \& Kagelmann 1993.

7 Vgl. statt anderer Diederichsen 2001 und die Beiträge in Bianchi 1996, 1997.

8 Vgl. statt anderer Altrogge 2000a, 2000b, 2000c, Richard 2001 und die Beiträge im dritten Kapitel von Neumann-Braun 1999.

9 Vgl. Bechdolf 2002, Kurp, Hauschild \& Wiese 2002 (S. 37-59), Rösing 2002 und Schwichtenberg 1992. 
Obwohl der Soziologe Klaus Neumann-Braun und seine Mitarbeiter einen ersten Überblick über die vorherrschenden Beobachtungen geben und somit die Komplexität der Diskursmöglichkeiten über Clips und Fernsehen dankenswerterweise reduzieren, fällt doch bereits an der analytischen Kategorisierung die mangelnde Trennschärfe auf. Für wen sind Clips Avantgarde und/oder Populärkultur? Wie sollen gesellschaftliche Kategorisierungen etwa von Zeitgeschichte gelöst betrachtet werden? Wie sollen Produktanalysen beobachterunabhängig (Stichwort: teilnehmende Beobachtung ohne teilzunehmen) stattfinden etc.?

Eine alternative Kategorisierungsmöglichkeit des Themenfeldes Musikclips nimmt der Soziologe Michael Altrogge (2000a: 5-7) in Anlehnung an einen Aufsatz des amerikanischen Kommunikationswissenschaftlers Joe Gow (1992) vor:

- Studien zur Geschichte von Musikvideos,

- inhaltsanalytische Studien zum Text Musikvideo,

- rezipientenorientierte Studien,

- kritische Studien.

Auch bei diesem Schema fallen thematische Überschneidungen auf, die eventuelle Codierungen im Rahmen von Meta-Analysen erschweren würden. Altrogge selbst modifiziert auch deshalb Gows Kategorien und betont die notwendige Fokussierung der Forschung auf den Zusammenhang von Bild und Ton und der damit einhergehenden Bedeutungskonstituierung auf drei Ebenen (vgl. Altrogge 2000: 19-20):

- Produktionsästhetische Strukturbestimmung,

- rezeptionsästhetische Fragestellungen,

- kommunikatunabhängige (externe) Funktionsbedingungen.

Für Altrogges Analyse des Zusammenspiels von Ton und Bild mag diese Kategorisierung sinnvoll erscheinen, einer übergreifenden Betrachtung von Musikclips, wie dies etwa jüngst auch Kathrin Fahlenbrach gefordert und vorbereitet hat (vgl. Fahlenbrach 2002), kann sie nur eingeschränkt dienen.

Eine gänzlich andere Kategorisierung schlägt der Kulturwissenschaftler Thomas Düllo (2000: 262) vor: Düllo betrachtet den Clip als Teil eines Gesamtkunstwerks »Popmusiker/Popmusikerin«, das durch drei wesentliche, rezipientenorientierte Leitdifferenzen bestimmt wird:

- Cool/heiß (uncool),

- natürliche/virtuelle Körperlichkeit,

- Künstlichkeit/Authentizität. 
Auch hier wird schnell klar, dass diese Kategorien auf die Analyse der Rezeption von Medienpersonen angewendet werden können, sie aber bei der Berücksichtigung des Gesamtbereichs Musikclip weite Felder unbearbeitet lassen müssen.

Die vier hier genannten möglichen Kategorisierungen zeigen das Dilemma des Forschungsfelds Musikclip exemplarisch: aus unterschiedlichen Disziplinen und Schulen wird sich mit verschiedenen Motiven und divergierenden Definitionen dem Untersuchungsgegenstand Musikclip genähert. Nur äußerst selten werden übergreifende Herangehensweisen erwähnt und umgesetzt. Zwar bleibt Altrogges überaus opulente Studie eine löbliche Ausnahme, eine, wie er selbst im Vorwort schreibt, »lange Reise « (Altrogge 2000a: V). Doch selbst dessen dreibändiger Versuch scheint letztlich - vom hohen Arbeitsaufwand einmal abgesehen ${ }^{10}$ - an Einzelfällen orientiert und monoperspektivisch bleiben zu müssen. Das belegt bereits ein erster Blick auf die Auswahl der dort untersuchten Clips. Aber warum sollten sich nicht eben ganze disziplinenübergreifende Forscherteams an solch faszinierende Zusammenhänge wie Clips und ihre Kontexte heranwagen, Repräsentativität erzeugen und am berüchtigten Puls der Zeit bleiben?

\section{Analyseebenen des massenkommunikativen Prozesses Musikclip und des Kompaktbegriffs Musikclip}

Um Neubaurs bereits erwähnte, berechtigte Kritik der Kontraproduktivität vieler Wissenschaftler wieder aufzunehmen und dazu beizutragen, dass diese in Bezug auf den Untersuchungsgegenstand Musikclips zumindest entkräftet wird, schlage ich eine eigene, an Siegfried J. Schmidts Ausführungen (1994, 2002) orientierte, analytische Kategorisierung des Bereichs Musikclip in Form des Massenkommunikationsprozesses Musikclip vor. ${ }^{11}$ Diese Kategorisierung offeriert eine komplexe Strukturierung des Problembereichs auf abstraktem Niveau, »um forschungspraktisch nötige Begrenzungen einschätzen, begründen und eventuell kompensieren zu können« (Schmidt 1994: 13).

Analysiert man auf dieser Folie den Gesamtbereich Musikclip in Deutschland, so sollte das System Musikclip in die von Schmidt für das Fernsehsystem der Bundesrepublik Deutschland heraus gearbeiteten Handlungsdimen-

10 Zwischen Einreichen und Publizieren seiner Studie benötigte Altrogge ca. vier Jahre.

11 Zur generellen Bedeutung des Prozessualen in der Popkulturanalyse und -praxis vgl. Hügel 2002, S. 64-68. 
sionen Produktion, Distribution, Rezeption und Weiterverarbeitung ${ }^{12}$ eingeteilt werden:

Produktion: Musiker, Künstler, Regisseure, Schauspieler etc.,

Distribution: Plattenfirmen, PR- bzw. Werbeagenturen, Musiksender, Kinos, Journalisten etc.,

Rezeption: Musikhörer, Fernsehzuschauer, Fans etc.,

Weiterverarbeitung: Fans, Journalisten, Kritiker.

In diesen Dimensionen wiederum handeln die beispielhaft aufgezählten Aktanten in Bezug auf die Medienangebote - hier Musikclips - in ganz bestimmten Rollen, die teilweise professionalisiert und institutionalisiert sind, »denn Medienangebote gibt es nur für Aktanten, und diese gehen immer in einer für sie je spezifischen Weise in konkreten Kontexten damit um; und Handlungen, die - im weitesten Sinne - auf Medienangebote konzentriert sind, beziehen sich in vielfältiger Weise aufeinander und beeinflussen sich gegenseitig « (Schmidt 1994: 13). Durch diese Einteilung wird m.E. ein strukturierter Überblick über den komplexen Gesamtzusammenhang Musikclip ermöglicht. Erst so kann forschungsökonomisch eingeschätzt und systematisch vorgegangen werden. Ferner lässt sich in diesem Schema eine sinnvolle Ausdifferenzierung des Rezeptions- als auch gesamten Kommunikationsprozesses erkennen, wie sie zuletzt Oliver Kautny (2002) speziell für Aspekte der musikalischen Rezeptionsgeschichte von Musik vorgetragen hat.

Eine zweite Einteilung scheint im Hinblick auf terminologische Klarheit bezüglich des Musikclips notwendig: Wenn Wissenschaftler von Musikclip als Medium reden, meinen sie manchmal die Bilder, manchmal das bereits erwähnte Kunstwerk, manchmal den Song oder die Band, selten aber den kompakten Gesamtzusammenhang Musikclip. ${ }^{13}$ Auch hier sei daher eine genaue Definition vorgeschlagen, angelehnt wiederum an Schmidt, der einen Medienkompaktbegriff als Systematisierungsinstrument mit vier, sich unter jeweils konkreten soziohistorischen Bedingungen bzw. Kontexten über soziale Handlungen selbst organisierenden Wirkungszusammenhängen beschrieben hat (vgl. Schmidt 2002: 56-57 bzw. Jacke/Jünger/Zurstiege 2000: 3233):

12 Schmidt schreibt von Verarbeitung, ich ergänze diese Handlungsdimension um das Präfix, um das Erstellen eines neuen Medienangebots durch den Rezipienten und den sich damit anschließenden, weiteren Kommunikationsprozess zu verdeutlichen.

13 Vgl. zu den divergierenden Begriffen grundsätzlich Hoffmann 1999. 
Kommunikationsinstrumente: hier die materialen Gegebenheiten Buchstaben (Schriften), Bildelemente (Gesamtbilder), Töne/Noten (Notationen, Partituren, Kompositionen),

Medientechnologien: hier Studio-, Tonträger- und zum Beispiel Filmtechnologien,

Sozialsystemische Komponenten: hier Organisationen wie Plattenfirmen, Distributionsfirmen (Vertriebe), Werbeagenturen, Musiksenderredaktionen etc.

Medienangebote: hier der eigentliche Musikclip, dessen Produktion, Distribution, Rezeption und Weiterverarbeitung kultürlich durch die drei anderen Faktorenbündel geprägt ist.

Im Grunde lassen sich diese beiden Bezugs- und Beobachtungsplattformen auch auf den Gesamtbereich Musik(industrie) ausdehnen. Doch hier geht es um die wissenschaftliche Behandlung speziell von Musikclips als >Mehr-alsMedienangeboten<. Schließlich werden Musikclips erst in komplexen Beziehungsgeflechten aus juristischen, ökonomischen, sozialen, politischen und anderen Kontexten beobacht- und analysierbar. Die Integriertheit des Clips als Medienangebot in solche kompakten Zusammenhänge und die Abhängigkeit von den anderen Ebenen und auch Prozessstufen sollte aber klar geworden sein.

\section{Transdisziplinäre Ausrichtung der Clipanalyse}

Begibt man sich als Medien- und Kommunikationswissenschaftler an den Untersuchungsgegenstand Musikclip und legt die beiden terminologischen Schablonen Kommunikationsprozess Musikclip und Kompaktbegriff Musikclip zu Grunde, dürfte eine erste Übersicht über mögliche zu analysierende Gebiete leichter fallen. Die folgenden exemplarischen Fragestellungen zu den beiden Einteilungen sollen genauer beleuchten, in welche disziplinäre Richtung die jeweiligen wissenschaftlichen Beobachtungen führen können und wie ahnungsloses bzw. »wildes Analysieren« (Neubaur 2002: 1083) vermieden werden kann: 


\subsection{Fragestellungen Massenkommunikationsprozess Musikclip}

Produktion:

Motive der Musiker,

Motive der Regisseure,

Überschneidungen der beiden Rollenmotive,

Bedeutungen weiterer Rollen für den Clip wie Künstler etc.,

Produktionsabläufe,

Produktionsästhetik,

ökonomische Aspekte der Produktion,

Distribution:

Motive der Vertriebe,

Bedeutung und Rolle der Werbung und PR,

Bedeutung und Rolle der institutionalisierten Musikmassenmedien (TV-

Sender etc.),

Konkurrenz auf den Märkten / ökonomische Aspekte,

Distributionsabläufe,

Distributionsästhetik,

ökonomische Aspekte der Distribution,

$\cdots$

Rezeption:

Motive der Rezipienten,

Gruppen von Rezipienten,

Rezeptionssituationen,

Rezeptionsabläufe,

Rezeptionsästhetik,

ökonomische Aspekte der Rezeption,

Verständnis der Clips und ihrer Elemente,

Weiterverarbeitung:

Bedeutung der Clips für Alltagsstrukturierungen und anschließendes Handeln,

Weiterverarbeitungsabläufe (Produktion von Anschluss-Medienangeboten),

Weiterverarbeitungsästhetik,

ökonomische Aspekte der Weiterverarbeitung,

Fankulturen,

...

Anhand dieser Themendimensionen und Fragefelder zeigt sich eine klare Analyselastigkeit in Richtung Medien-, Kommunikations- und Kulturwissenschaft. Aber sicherlich dürften auch Wissenschaftler angrenzender Bereiche wie etwa Musik- und Kunstwissenschaft, Psychologie, Pädagogik und Soziologie ganz besondere Interessen an den Prozessstufen des Musikclips haben und ihre eigenen Theorien und Methoden in die Analysen mit einbringen. Im Grunde lassen sich so innerhalb der Dimensionen und anhand der dort zu 
bearbeitenden Fragefelder transdisziplinäre Forscherteams zusammenstellen und somit ganz neue Wissenschaftsgruppierungen zu einem Thema konstituieren.

\subsection{Fragestellungen Kompaktbegriff Musikclip}

Kommunikationsinstrumente:

Analyse von Bildelementen und deren Bedeutung für das Gesamtbild des Clips,

Analyse von Tonelementen und deren Bedeutung für den Klang eines Clips, Analyse von Schriftelementen (z.B. Slogans) und deren Bedeutung für die

Gesamtschrift bzw. -text Clip, $\ldots$

Medientechnologien:

Bedeutung unterschiedlicher Technologien für die Produktion, Distribution, Rezeption und Weiterverarbeitung von Clips,

Kommunikationsvoraussetzungen bzw. -barrieren durch Technologien,

$\ldots$

Sozialsystemische Komponenten:

Analysen von Filmteams, Redaktionen, Labels, Vertrieben etc.,

Vermarktungs- und musikwirtschaftliche Untersuchungen,

Medienangebote:

Analyse des gesamten Medienangebots Clip (Berücksichtigung aller unter Kommunikationsinstrumente genannten Elemente),

Ästhetik der Clips,

Platzierung der Medienangebote im Programmfluss (Flow),

Programmanalyse,

Anhand dieser Themendimensionen und Fragefelder zu den einzelnen Komponenten des Kompaktbegriffs Musikclip wird deutlich, was gemeint sein und analysiert werden kann, wenn Wissenschaftler von Clips oder vom Medium Musikclip reden. Hier zeigt sich ebenfalls, an welchen Stellen etwa Kunst-, Musik-, Medien-, Wirtschafts- oder Kulturwissenschaftler mit ihrem Spezialwerkzeug ansetzen können. Und genau an diesen Fragestellungen wird deutlich, wie sehr eine nicht nur inter-, sondern vielmehr transdisziplinäre - also durch die Fächer hindurch laufende - Analyse zum Beispiel im Rahmen von größeren Forschungsprojekten vonnöten scheint. Diese transdisziplinären Ausrichtungen einer Clipanalyse, diese Methoden- und Theoriedialoge im Sinne von Helmut Schanze (2002) sollten bereits in der Ausbildung, etwa in den immer noch viel zu selten stattfindenden Seminaren der Hoch- und Fachhochschulen beginnen. 
Ein Beispiel: In einer eigenen Lehrveranstaltung zur Musikclipanalyse im Sommersemester 2002 konnten unterschiedliche disziplinäre Vor-Ausbildungen überaus fruchtbar transdisziplinär genutzt werden - Synergie- und Lerneffekte auch für den Dozenten inbegriffen. So wurden, nach einigen theoretischen Vorüberlegungen und der intensiven Diskussion verschiedener Clip-Bereiche (Clip und Alptraum, Clip und Bild, Clip und Rhythmus etc.), übergreifende medien-, wirtschafts-, geschichts- und insbesondere musikwissenschaftliche Herangehensweisen exemplarisch an Clips von Bob Dylan, Bon Jovi oder Morrissey erprobt und über den Zusammenhang zwischen Kunst und Werbung als gesellschaftlichem Zuordnungsbereich für Musikclips diskutiert. In den an die Veranstaltung anschließenden Leitfadeninterviews mit den Seminarteilnehmern wurde nochmals deutlich, wie fruchtbar die aus diversen Fächern zusammengetragenen Erfahrungen für dieses Seminar waren und wie sehr ein intensiverer, offenerer und übergreifenderer Zugang zu popkulturellen und alltäglich omnipräsenten Phänomenen wie Musikclips in einer Medienkulturwissenschaft, aber auch in anderen Disziplinen seitens der Studierenden gewünscht wird. ${ }^{14}$

\section{Fazit}

»Internet Killed the Video Star?« Keinesfalls! Auch wenn die von mir befragten Studierenden das Musikfernsehen und auch das Internet eher als Nebenbeimedium für den Musikkonsum nutzen, so scheinen sich die zeitlich aufeinander folgenden Medientechnologien vielmehr zu ergänzen, denn zu verdrängen. Bemerkenswert bleibt die Bedeutung von Musikclips zur Information und vor allem Illustration von popmusikalischen Stücken. Und auch wenn die Interviews und zahlreichen, darüber hinaus reichenden Gespräche zu dieser Problematik nicht repräsentativ sein können, so wird doch klar, dass das Interesse und die Sensibilität an der Analyse popkultureller Medienangebote weiter wächst und vor allem ein Strukturierungsbedarf der Problembereiche besteht - und zwar über so genannte Fachgrenzen hinaus.

Eine solche transdisziplinäre Vorstrukturierung habe ich für den Massenkommunikationsprozess Musikclip und den Kompaktbegriff Musikclip vorgeschlagen, auch, um die bereits 1997 von Winfried Pape und Kai Thomsen eingeforderte Multiperspektivität aus medien- und musikwissenschaftlichen Positionen bei der Clipanalyse einzulösen. Um die löbliche Ausnahme Mi-

14 Die Leitfäden wie auch die zehn auf $M D$ aufgezeichneten, ca. 45minütigen, qualitativ und mit zumeist offenen Fragen ausgerichteten Interviews sind beim Autor einzusehen. 
chael Altrogge (2002a: 3) nochmals zu Wort kommen zu lassen: »Welche Wissenschaftsdisziplin sich auch beteiligt - die Welt der Musikvideos dient dazu, die unterschiedlichsten Hypothesen zu illustrieren. « Warum sollte man diese Hypothesen und ihre transdisziplinäre Überprüfung nicht weiter systematisieren?

\section{Literatur}

Altrogge, Michael (2000a). Tönende Bilder. Interdisziplinäre Studie zu Musik und Bildern in Videoclips und ihrer Bedeutung für Jugendliche. Bd. 1: Das Feld und die Theorie. Berlin: Vistas.

Altrogge, Michael (2000b). Tönende Bilder. Interdisziplinäre Studie zu Musik und Bildern in Videoclips und ihrer Bedeutung für Jugendliche. Bd. 2: Das Material: Die Musikvideos. Berlin: Vistas.

Altrogge, Michael (2000c). Tönende Bilder. Interdisziplinäre Studie zu Musik und Bildern in Videoclips und ihrer Bedeutung für Jugendliche. Bd. 3: Die Rezeption: Strukturen der Wahrnehmung. Berlin: Vistas.

Bechdolf, Ute (2002). »Puzzling Gender - Jugendliche verhandeln Geschlecht im und beim Musikfernsehen. «In: Wozu Jugendliche Musik und Medien gebrauchen. Jugendliche Identität und musikalische und mediale Geschmacksbildung. Hg. v. Renate Müller, Patrick Glogner, Stefanie Rhein und Jens Heim. Weinheim und München: Juventa, S. 222-230.

Bianchi, Paolo (Hg.) (1996a). Art \& Pop \& Crossover (= Kunstforum International Bd. 134). Ruppichteroth: Kunstforum-Bücherdienst.

Bianchi, Paolo (Hg.) (1996b). Cool Club Cultures (= Kunstforum International Bd. 135). Ruppichteroth: Kunstforum-Bücherdienst.

Bódy, Veruschka / Weibel, Peter (Hg.) (1987). Clip, Klapp, Bum. Von der visuellen Musik zum Musikvideo. Köln: DuMont.

Diederichsen, Diedrich (2000). »Die Politik der Aufmerksamkeit. Visual Culture, Netzkunst und die Unterscheidung von Kunst und Nichtkunst.« In: Imagineering. Visuelle Kultur und Politik der Sichtbarkeit. Hg. v. Tom Holert. Köln: Oktagon, S. 70-84.

Düllo, Thomas (2000). »Coole Körpermaschinen, hysterisierte Räume. Maskierte Identitätsvokabeln in neueren Musik-Clips. « In: Kursbuch Kulturwissenschaft. Hg. v. Thomas Düllo, Arno Meteling, André Suhr und Carsten Winter. Münster u.a.: Lit, S. 259-275.

Fahlenbrach, Kathrin (2002). Feeling Sounds: Emotional Aspects of Music Videos. Halle-Wittenberg: Manuskript [http://www.arts.ualberta.ca/igel/IGEL2002/ Fahlenbrach.pdf; Download 20.12.2002].

Gass, Lars Henrik (2001). »Dekontextualisierungen. Was hat Popkultur mit (Kurz-) Film zu tun? « In: Pop Unlimited? Imagetransfers in der aktuellen Popkultur. Hg. v. Christian Höller. Wien: Turia u. Kant, S. 75-91.

Gow, Joe (1992). »Making Sense of Music Video: Research During an Inaugural Decade. « In: Journal of American Culture 15, Nr. 3, S. 35-43.

Hoffmann, Justin (1999). »Das Musikvideo als ökonomische Strategie.« In: Das Phantom sucht seinen Mörder. Ein Reader zur Kulturalisierung der Ökonomie. Hg. v. Justin Hoffmann und Marion von Osten. Berlin: b_books, S. 65-75. 
Hügel, Hans-Otto (2002). »Zugangsweisen zur Populären Kultur. Zu ihrer ästhetischen Begründung und zu ihrer Erforschung. «In: Populäre Kultur als repräsentative Kultur. Die Herausforderung der Cultural Studies. Hg. v. Udo Göttlich, Winfried Gebhardt und Clemens Albrecht. Köln: Herbert von Halem, S. 52-78.

Jacke, Christoph / Jünger, Sebastian / Zurstiege, Guido (2000). »Aufdringliche Geschichten - Zum Verhältnis von Musik und Werbung.«In: Populäre Musik im kulturwissenschaftlichen Diskurs. Hg. v. Helmut Rösing und Thomas Phleps (= Beiträge zur Popularmusikforschung 25/26). Karben: Coda, S. 25-42.

Kautny, Oliver (2002). »Musikalische Rezeptionsgeschichte: Die Differenz zwischen Wahrnehmung und Kommunikation. «In: Musik \& Ästhetik 6, H. 23, S. 46-60.

Kothenschulte, Daniel (2002). »Opas Video ist tot. Vom Bedeutungszwang befreit: Musikclips bei den Oberhausener Kurzfilmtagen. «In: Frankfurter Rundschau, Nr. 105 (7. Mai), S. 23.

Kurp, Matthias / Hauschild, Claudia / Wiese, Klemens (2002). Musikfernsehen in Deutschland. Politische, soziologische und medienökonomische Aspekte. Wiesbaden: Westdeutscher.

Langhoff, Thomas (1999). »Video Killed the Radio Star. MTV und Clip-Kultur.«In: »alles so schön bunt hier«. Die Geschichte der Popkultur von den Fünfzigern bis heute. Hg. v. Peter Kemper, Thomas Langhoff und Ulrich Sonnenschein. Stuttgart: Philipp Reclam jun., S. 228-240.

Magdanz, Fee (2001): »Prêt-à-Pop. Stoff-Fetzen - Fragmente einer Geschichte von Maskerade und Pop.«In: Sound Signatures. Pop-Splitter. Hg. v. Jochen Bonz. Frankfurt/M.: Suhrkamp, S. 131-140.

Morgenroth, Gisbert (1994). "Geschichte der visuellen Musik.« In: Handbuch der Musikwirtschaft. Hg. v. Rolf Moser und Andreas Scheuermann. Starnberg, München: Josef Keller (3. Aufl.), S. 287-294.

Neubaur, Caroline (2002). »Das Bild - Patient oder Kollege? Zur Psychoanalyse der bildenden Kunst.« In: Merkur, Deutsche Zeitschrift für europäisches Denken, H. 644, S. 1079-1090.

Neumann-Braun, Klaus / Barth, Michael / Schmidt, Axel (1997). "Kunsthalle und Supermarkt - Videoclips und Musikfernsehen. « In: Rundfunk und Fernsehen 45, Nr. 1, S. 69-86.

Neumann-Braun, Klaus / Schmidt, Axel (1999). »McMusic. Einführung.« In: VIVA MTV! Popmusik im Fernsehen. Hg. v. Klaus Neumann-Braun. Frankfurt/M.: Suhrkamp, S. 7-42.

Pape, Winfried / Thomsen, Kai (1997). "Zur Problematik der Analyse von Videoclips. «In: Step across the Border. Neue musikalische Trends - neue massenmediale Kontexte. Hg. v. Helmut Rösing (= Beiträge zur Popularmusikforschung 19/20). Karben: Coda, S. 200-226.

Richard, Birgit (2001). »Bild-Klone und Doppelgänger. Vervielfältigungsmechanismen in der Popkultur.« In: Transgene Kunst: Klone und Mutanten. Hg. v. Birgit Richard und Sven Drühl (= Kunstforum International Bd. 157). Ruppichteroth: Kunstforum-Bücherdienst, S. 55-111.

Rösing, Helmut (2002). »Populäre Musik und kulturelle Identität. Acht Thesen.«In: Heimatlose Klänge? Regionale Musiklandschaften - heute. Hg. v. Thomas Phleps (= Beiträge zur Popularmusikforschung 29/30). Karben: Coda, S. 11-34.

Schanze, Helmut (2002). »Medien, Methoden, Theorien. Nachüberlegungen zum Methoden- und Theoriedialog des Sonderforschungsbereichs 240 >Bildschirmmedien «.« In: Bildschirm - Medien - Theorien. Hg. v. Peter Gendolla, Peter Ludes und Volker Roloff. München: Wilhelm Fink, S. 23-32. 
Schmidt, Siegfried J. (1994). »Einleitung: Handlungsrollen im Fernsehsystem. « In: Vom »Autor« zum Nutzer: Handlungsrollen im Fernsehen. Hg. v. Werner Faulstich. München: Fink, S. 13-26.

Schmidt, Siegfried J. (2002). »Medienwissenschaft im Verhältnis zu Nachbardisziplinen. « In: Einführung in die Medienwissenschaft. Konzeptionen, Theorien, Methoden, Anwendungen. Hg. v. Gebhard Rusch. Wiesbaden: Westdeutscher, S. 53-68.

Schwichtenberg, Cathy (1992). »Music Video: The Popular Pleasures of Visual Music. «In: Popular Music and Communication. Hg. v. James Lull. Newbury Park u. a.: Sage (2. Aufl.), S. 116-133.

Statistisches Bundesamt (Hg.) (2002). Datenreport 2002. Zahlen und Fakten über die Bundesrepublik Deutschland. Bonn: Bundeszentrale für politische Bildung.

Weibel, Peter (1987a). »Von der visuellen Musik zum Musikvideo.«In: Bódy/Weibel 1987: 53-163.

Weibel, Peter (1987b). »Was ist ein Videoclip?« In: Bódy/Weibel 1987: 274-275.

Winter, Rainer; Kagelmann, H. Jürgen (1993). »Videoclip.« In: Musikpsychologie. Ein Handbuch. Hg. v. Herbert Bruhn, Rolf Oerter und Helmut Rösing. Reinbek: Rowohlt, S. 208-220.

\begin{abstract}
On the one hand, in the late 1990s in newspapers and special interest magazines as well as in scientific publications discussions about music videos have decreased in numbers. Regarding this fact it seems interesting that - on the other hand - especially because of new media technologies such as the internet and DVD there can be observed a new kind of technical availability of music clips (»clip« here means short music film, not only based on video technology). Moreover, there is a new generation of scholars and intellectuals growing up who are open minded towards the use and role of popular media culture (as materialized in form of music clips) for everyday life. Obviously a new scholarship slowly develops from the temporarily silence of media culture societies. My paper focuses on the complex communicative process of music clips as well as on the complex concept of music clips to show a way to categorise this topic and group different dimensions to analyse clips. Consequently, this should lead to more structured and transdisciplinary approaches within the above mentioned new scholarship.
\end{abstract}

